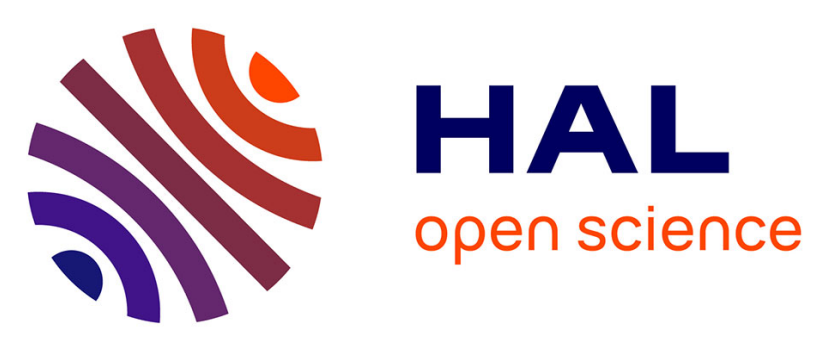

\title{
A DFT/TD-DFT study for the ground and excited states of peramine and some pyrrolopyrazinone compounds
}

\author{
Andrzej Lapinski, Alina Teresa Dubis
}

\section{- To cite this version:}

Andrzej Lapinski, Alina Teresa Dubis. A DFT/TD-DFT study for the ground and excited states of peramine and some pyrrolopyrazinone compounds. Journal of Physical Organic Chemistry, 2009, 22 (11), pp.1058-n/a. 10.1002/poc.1558 . hal-00477800

\section{HAL Id: hal-00477800 \\ https://hal.science/hal-00477800}

Submitted on 30 Apr 2010

HAL is a multi-disciplinary open access archive for the deposit and dissemination of scientific research documents, whether they are published or not. The documents may come from teaching and research institutions in France or abroad, or from public or private research centers.
L'archive ouverte pluridisciplinaire HAL, est destinée au dépôt et à la diffusion de documents scientifiques de niveau recherche, publiés ou non, émanant des établissements d'enseignement et de recherche français ou étrangers, des laboratoires publics ou privés. 


\section{Journal of Physical Organic Chemistry}

WILEY

\section{A DFT/TD-DFT study for the ground and excited states of peramine and some pyrrolopyrazinone compounds}

\begin{tabular}{|r|l|}
\hline Journal: & Journal of Physical Organic Chemistry \\
\hline Manuscript ID: & POC-08-0193.R3 \\
\hline Wiley - Manuscript type: & Research Article \\
\hline Date Submitted by the \\
Author: & 03-Mar-2009 \\
\hline Complete List of Authors: & $\begin{array}{l}\text { Lapinski, Andrzej; Institute of Molecular Physics, Polish Academy of } \\
\text { Sciences } \\
\text { Dubis, Alina; Institute of Chemistry, University of Bialystok }\end{array}$ \\
\hline Keywords: & $\begin{array}{l}\text { peramine, antifeedants, DFT and TD-DFT calculations, Raman } \\
\text { spectroscopy, electronic spectra }\end{array}$ \\
\hline \multicolumn{2}{|c}{} \\
\hline
\end{tabular}

\section{s scholaroNE" \\ Manuscript Central}




\title{
A DFT/TD-DFT study for the ground and excited states of
}

\section{peramine and some pyrrolopyrazinone compounds}

\author{
Andrzej Lapiński ${ }^{a}{ }^{*}$ and Alina T. Dubis ${ }^{b}$ \\ ${ }^{a}$ Institute of Molecular Physics, Polish Academy of Sciences, ul. Smoluchowskiego 17, \\ 60-179 Poznan, Poland \\ ${ }^{b}$ Institute of Chemistry, University of Bialystok, Al. Pilsudskiego11/4, 15-443 Bialystok, \\ Poland
}

\begin{abstract}
Peramine, a heterocyclic natural molecule, reveals two main, different in nature, electronic absorption bands. The theoretical calculations at TD-B3LYP/6$311++G(d, p)$ level of theory show that the electronic excitations are connected predominantly with $\pi \rightarrow \pi^{*}$ and charge-transfer (CT) transitions. Excitation of electrons from the pyrrolopyrazinone ring to the side chain plays the role in creating CT transition. The character and energy of the first 30 singlet-singlet electronic transitions have been also investigated for the most stable conformation of peramine.
\end{abstract}

Keywords: peramine; antifeedants; DFT and TD-DFT calculations; electronic spectra, Raman spectroscopy;

*Corresponding author. Tel.: +48-61-869-5279; fax: +48-61-868-4524

E-mail address: lapinski@ifmpan.poznan.pl (A. Łapiński) 


\section{INTRODUCTION}

Peramine

3-(1,2-dihydro-2-methyl-1-oxopyrrolo[1,2-a]pyrazon-3yl)propyl)guanidine) (1) is a heterocyclic natural product and belongs to the class of alkaloids with pyrrolopyrazinone substructure. It has been isolated from plants and identified as secondary metabolite protecting plant against grazing by insects. ${ }^{[1,2,3,4,5,6,7]}$ Some other propyl-substituted derivatives of peramine (1) as (3-(3-aminopropyl)-2methylpyrrolo[1,2-a]pyrazin-1-(2H)-one) (2), (3-(3-chloropropyl)-2-methylpyrrolo[1,2a]pyrazin-1-(2H)-one) (3), and (3-propyl-2-methylpyrrolo[1,2-a]pyrazin-1-(2H)-one) (4) act as antifeedant agents as well. ${ }^{[8]}$ Peramine (1) has received a great deal of attention during the years ${ }^{[9,10,11,12]}$ because of its potential application as synthetic biopesticide. It may also serve as a template for the design of the new feeding inhibitors. However, scientific data published to date do not extend beyond description of the biological properties of peramine and methods of its isolation and preparation. For the sake of completion of the neglected spectral data we subjected peramine to the spectroscopic and theoretical investigations. This paper is a continuation and extension of our previous study on vibrational properties of the pyrrolopyrazinone compounds 14. ${ }^{[13]}$ In the present work the electronic absorption spectra of peramine were studied. The spectra were recorded for solid state sample and for solution sample as well. Such methods of the analysis were chosen because secondary metabolites as a peramine usually occur in aqueous microenvironment of the plant tissues. There is a reason to believe that some rotational forms may occur under the circumstances. We also decided to approach the problem theoretically. Theoretical calculations have been applied to examine the geometries as well as the energies of the possible conformations. Furthermore, for the description of the excited states and optical spectra of peramine, 


\section{EXPERIMENTAL AND METHODOLOGY}

Peramine and other pyrrolopyrazinone derivatives were synthesized by Dumas method; the detailed information about synthesis is available elsewhere. ${ }^{[8]}$ The vibrational spectra of 1-4 were investigated at room temperature for polycrystalline samples using Raman scattering technique. The spectra in the range from 50 to 4000 $\mathrm{cm}^{-1}$ were investigated with a Jobin Yvon HORIBA LabRAM HR 800 confocal spectrometer with a liquid- $\mathrm{N}_{2}$-cooled charge couple device (CCD) and two lasers: Stabilite 2017 argon ion laser $\left(\lambda_{\text {laser }}=514,488 \mathrm{~nm}\right)$ and He-Ne $\left(\lambda_{\text {laser }}=633 \mathrm{~nm}\right)$ and using a Renishaw System 1000 microspectrometer with excitation beam from laser Lexel $3500\left(\lambda_{\text {laser }}=568 \mathrm{~nm}\right)$ within spectral range from 300 to $4000 \mathrm{~cm}^{-1}$. The laser power before focusing with objective was less than $10 \mathrm{~mW}$. The spectral resolution of Raman spectra was $2 \mathrm{~cm}^{-1}$. Additionally, we studied the absorption electronic spectra of 
investigated compounds in $\mathrm{KBr}$ pellets and in solutions in the spectral region from 3200 to $50000 \mathrm{~cm}^{-1}$ using Perkin - Elmer UV/Vis/NIR Lambda 19 spectrometer.

In order to interpret intramolecular excitations in the electronic spectra and vibrational transitions we carried out quantum-chemical calculations, which have been performed following a two-step procedure: the optimization of the ground-state geometry with B3LYP and PBE0 theory levels using a basis set 6-311++G(d,p) and the determination of the electronic transition energies by means of TD-B3LYP/6$311++\mathrm{G}(\mathrm{d}, \mathrm{p})$ and TD-PBE0/6-311++G(d,p). ${ }^{[17,18,19]}$ We computed the first 30 singletsinglet electronic transitions by applying the time-dependent DFT approximation ${ }^{[20]}$ to their correspondingly optimized ground state geometries. Calculations of molecules 1-4 were made on a single molecule and in ethanol (Tomasi's PCM Model) using Gaussian 03 program package. ${ }^{[21]}$ The vibrational transitions were performed at B3LYP theory level using a basis set $6-311++\mathrm{G}(\mathrm{d}, \mathrm{p})$ where the B3LYP functional includes a mixture of HF exchange with DFT exchange-correlation, given by Becke's three-parameter functional $^{[22,23]}$ with the Lee, Yang, and Parr correlation functional. ${ }^{[24]}$ The computed frequencies were scaled using the factor 0.98 to eliminate known systematic errors, correcting the anharmonicity. ${ }^{[25]}$ The mode description was performed by visual inspection of the individual modes using the GaussView program. Moreover, the theoretical Raman intensities were calculated from scattering activities and calculated wavenumbers obtained from the Gaussian calculations. ${ }^{[26,27,28]}$

In our investigations three conformations of investigated compounds have been taken into account. The first one is the flat conformation where aliphatic chain lies in the same plane as pyrrolopyrazinone rings and the other two are the bent conformations 
where this chain is located out of rings plane due to $\mathrm{sp}^{3}$ hybridization of carbon atoms in $\mathrm{R}$ side chain.

\section{RESULTS AND DISCUSSION}

\section{Geometrical and energetical parameters of peramine}

Recently, we have found on the basis of theoretical and IR and Raman investigations, that peramine derivatives exist in flat form in solid state. ${ }^{[13]}$ This conclusion was also confirmed by the X-ray based investigation of the crystal structure. ${ }^{[13]}$ It was also found that difference in energy between the flat and bent rotameric forms is rather small and the bent conformation in gas phase is more stable than the flat form. Hence, the process of transformation of the flat form into the bent one is investigated here.

Fig. 1 shows the dependence between the energy of peramine molecule and the angle between the planes: pyrrolopyrazinone ring and the side chain. In the Fig. 2 the dihedral angle was defined as an angle $\tau(\mathrm{C} 10, \mathrm{C} 13, \mathrm{C} 15, \mathrm{C} 17)$. Fig. 3 presents the flat conformer (II) where the plane of the side chain lies in the same plane as pyrrolopyrazinone rings and the bent conformers (I and III) where these planes are twisted in respect to each other. The calculated geometrical parameters of the rotameric forms I, II, and III of peramine are presented in the Table 1. There are no significant differences between the bond lengths of the studied conformers I-III. The geometry of the peramine rings is planar and the side chain plane may be twisted in respect to rings. The angle $\tau$ (Fig. 2) equals $1.6^{\circ}$ and $95.8^{\circ}, 276.3^{\circ}$ for planar and bent conformations, respectively.

[Insert Figs 1 and 2 about here.] 
The resulting optimized geometry of rotameric states I, II, III and transition states TS1, TS2, TS3 are shown in Fig. 3. TS1 connects I and III, TS2 connects III and II, and TS3 connects II and I forms. The more stable conformation I is assumed here as the reference zero energy level (Table 2). The barrier height for the transformation of the I conformation into the II and III amount to 2.95 and $2.94 \mathrm{kcal} / \mathrm{mol}$, respectively. The barrier height for the transformation from I to III conformation (TS1) amounts 50.03 $\mathrm{kcal} / \mathrm{mol}$. The conformation TS1 of peramine corresponds to the structure with the dihedral angle $\tau$ equals $10^{\circ}$. The relatively large barrier height may hinder interconversion between I and III state. On the other, hand barrier heights can decrease with increasing solvent polarity due to push-pull character of compounds. ${ }^{[29,30,31,32]}$ Table 2 shows also the dipolar moment values of peramine state I, II, and III. The highest value was observed for flat conformation II, whereas the lowest dipolar moment was observed for TS1 transition state.

\section{[Insert Fig. 3 about here.]}

Furthermore, the geometry of compounds $\mathbf{2 - 4}$ was also studied. Table 3 reveals that the bent form is energetically more stable than the flat conformation. The selected geometrical parameters as the bond distances and dihedral angles for more stable geometries of the species under study (1-4) are summarized in Table 4. One may see that the geometry of the pyrrolopyrazinone rings for all investigated compounds is planar and most of the calculated bond lengths are the same. This observation indicates that the nature of the side chain (R-substituents) have no significant influence on geometrical parameters of the pyrrolopyrazinone ring.

\section{Vibrational properties}


The infrared and Raman analysis supported by DFT calculations were performed for peramine (1). Results of these experiments are presented in Fig. 4. Raman spectra of peramine were recorded for polycrystalline sample for different excitation lines and compared with the calculated spectra of the rotamers I, II, and III.

[Insert Fig. 4 about here.]

The stable conformations (I-III) were found with support of DFT calculations. Calculated data showed no imaginary frequencies, so the energy minima were true. The detailed assignments of normal modes of peramine and its derivatives were done by us previously and presented elsewhere. ${ }^{[13]}$ In the spectral region presented in Fig. 4 the most intensive bands are observed at $1527,1379,1360$, and $1335 \mathrm{~cm}^{-1}$. The first mode is related to the stretching $\mathrm{C}=\mathrm{C}$ bonds within pyrrole ring. The corresponding calculated frequency is in very good agreement with the experimental data. Moreover, this band is located at the similar position at 1533, 1530, and $1532 \mathrm{~cm}^{-1}$ for compounds 2-4. A good correlation for this mode was also observed for both IR calculated and experimental data. The other bands $\left(1379,1360\right.$, and $\left.1335 \mathrm{~cm}^{-1}\right)$ are due to carbon-nitrogen stretching vibration of the aromatic systems. Similarly, for compounds 2-4 the position of these bands are present in the same spectral region. For example, the band $1360 \mathrm{~cm}^{-1}$ for peramine is observed at 1366, 1368, and $1364 \mathrm{~cm}^{-1}$ for $\mathbf{2}, \mathbf{3}$, and 4 molecules, respectively. Generally speaking, structural modification of the side chain (compounds 2-4) do not significantly influence the position of $v_{\mathrm{C}-\mathrm{N}}$ pyrrole ring vibrational modes. However, the intensity and position of the calculated modes are dependent on the type of conformation (see Fig. 4). From these observations we suggest that $v_{\mathrm{C}-\mathrm{N}}$ pyrrole stretching bands may be applied as diagnostic features for recognition of rotameric form. In order to be sure that the intensities of these bands are independent from the 
excitation energy of light we have also analyzed the Raman spectra for four different laser excitation lines (see Fig. 4). No significant intensity changes in experimental spectra were observed. It leads us to conclude that the resonance Raman scattering effect is not present for peramine.

Moreover, previously performed structural investigations ${ }^{[13]}$ constitute the final proof of the rotameric form presented in solid state. Based on the crystal data it was concluded that there is only a flat form in solids. Considering the results of investigations we can suggest that some other factors as steric hindrance and intermolecular interactions ${ }^{[33,34,35]}$ inhibit conformer interconversion process into energetically more stable bent form (I).

\section{Electronic energies}

Table 5 presents the energies of the frontier orbitals of three rotameric forms of peramine (I, II, III). One can see that eigenvalues of our systems depends on the conformational form. The calculated energies differ in most cases by less than $0.1 \mathrm{eV}$. Our study enable us to suggest that the HOMO, HOMO-1, HOMO-3, LUMO, LUMO+2, LUMO+3 orbitals are localized on pyrrolopyrazinone rings, whereas HOMO-2, HOMO-4, LUMO+1 are localized on aliphatic chain. The visualization of some molecular orbitals is presented in Fig. 5.

[Insert Fig. 5 about here.]

Table 6 presents the eigenvalues of molecules 1-4 corresponding to the highest occupied (HOMO) and lowest unoccupied (LUMO) molecular orbitals. The HOMOLUMO energy gap amounts 4.93, 4.90, 4.95, and $4.90 \mathrm{eV}$ for compound $\mathbf{1}, \mathbf{2}, \mathbf{3}$, and 4, respectively. The LUMO level energy range of peramine systems is 0.92 to $0.79 \mathrm{eV}$ and 


\section{Electronic spectra}

Figs 6 and 7 show the absorption spectra recorded for peramine (1) and its derivatives (2-4), respectively. Spectra were taken in water and ethanol solutions and for polycrystalline samples. The spectra consist of two broad and strong bands. The absorption maxima are observed at about $34500 \mathrm{~cm}^{-1}(4.3 \mathrm{eV})$ (band A) and at about $43500 \mathrm{~cm}^{-1}(5.4 \mathrm{eV})$ (band $\left.\mathrm{B}\right)$. The position of electronic transitions presented in Table 7 was determined by fitting the spectra using Gaussian curves; the quality of fits is shown in Fig. 6.

\section{[Insert Figs 6 and 7 about here.]}

For the interpretation of experimental data we have applied TD-DFT. This theory has become lately one of the more accurate methods for calculating excitation energies of molecules, clusters, and solids. The calculations yield transition frequencies with errors below $0.2 \mathrm{eV}^{[36]}$ In some cases ${ }^{[37,38,39,40,41]}$, there are some limitations of TD-DFT which overestimate the electronic transitions up to $1 \mathrm{eV} .^{[42,43]}$

In Table 7 and in Figs 6 and 7 (lowest panels) excitation energies $(\Delta E$ in $\mathrm{eV})$ of singlet-singlet transitions and oscillator strengths $f$ of the excited states of peramine (1) and chloro derivative (3) for bent and flat conformations are presented. Theoretically calculated data for peramine were compared with experimental electronic spectra (Fig. 6). Our theoretical results were consistent with the experimental data. The band A is a predominantly $\pi-\pi^{*}$ type involving $\mathrm{HO}$ and LU delocalized $\pi$ orbitals of the 
pyrrolopyrazinone rings and this transition is connected with excitation from HOMO to LUMO and from HOMO to LUMO+2 (Fig. 5). The band B is the superposition of transitions: from HOMO to LUMO+7, from HOMO to LUMO+8, from HOMO-1 to LUMO, and from HOMO to LUMO+2 (Fig. 5). Considering the results, we can suggest that the charge-transfer $(\mathrm{CT})$ and $\pi-\pi^{*}$ transitions play the largest role in creating this band. The CT process involves the excitation of electrons from the pyrrolopyrazinone rings to the side chain. We can state that bands A and B are different by nature.

Moreover, the sensitivity of the absorption spectra of peramine and its derivatives to medium polarity has been observed (Table 7). Absorption bands A and B detected for peramine in solutions are shifted in comparison with solid state spectra. The band $\mathrm{A}$ is blue shifted by 0.05 and $0.06 \mathrm{eV}$ for water and ethanol solutions, respectively, whereas, the band $\mathrm{B}$ is red shifted by $0.03 \mathrm{eV}$. These red and blue shifts can be classified as solvatochromic shifts. ${ }^{[44]}$ These phenomena are attributed to specific chemical effects of the solvent on electronic states. Such specific effects as hydrogenbond formation, proton or charge transfer between solvent and solute, solventdependent aggregation, ionization or isomerization equilibria can play a crucial role in these phenomena. The other category of shifts is related to physical interactions between the solute and solvent molecules. In such a case the electric moments of the solute lead to reflection moments in the solvents that are aligned to give an attractive interaction, reducing the energy of the solute. For investigated compound 1-4 the effect of polarization of the solvent continuum (TD-PBE0/6-311++G(d,p) Tomasi's PCM model $)^{[45,46,47,48]}$ was taken into account and the results are presented in Table 7.

In experimental spectra (Figs 6 and 7) one can find that solvent has two main effects: absorption intensity was changed and position of bands was shifted depending 
on type of solvent. These effects have been investigated by Scott et al.. ${ }^{[49]}$ They have shown that these effects are related to phenomenon of mixing of electronic states of the solute under the influence of the solvent which must have proper orientation to couple the solute states. Moreover, it was shown that the interaction in one solvent-solute pair accounts for most of the borrowed intensity and the largest perturbation occurs when the solvent and solute are in contact. It seems most likely that observed shift phenomena for peramine derivatives 1-4 can be attributed to effects related to physical interactions between the solute and polar solvents as water and ethanol and to the intermolecular interactions as a hydrogen bonds. ${ }^{[50,51]}$

\section{CONCLUSIONS}

In conclusion we can state that we have performed interpretation of electronic spectra of peramine and its derivatives. We have shown that the electron density distribution on peramine atoms for HOMO and LUMO are only observed in the planar part of the molecule. In electronic spectra of the investigated compounds the band $\mathrm{A}$ is observed at about $4.3 \mathrm{eV}$ and band $\mathrm{B}$ at about $5.4 \mathrm{eV}$. These bands are related to the $\pi$ $\pi^{*}$ transition and to the superposition of $\pi-\pi^{*}$ and charge-transfer ones, respectively. Electronic absorption bands showed solvatochromic shifts in polar solvent. A satisfying experiment/theory agreement was reached by applying TD-DFT method of calculation.

\section{Acknowledgements}

This work was supported by the Polish Committee for Scientific Research at the research project in 2006-2008.

\section{REFERENCES}


[1] D.D. Rowan, D.L. Gaynor, J. Chem. Ecol. 1986, 12, 647-658.

[2] M.R. Siegel, G.C.M. Latch, L.P. Bush, F.F. Fannin, D.D. Rowan, B.A.Tapper, C.W. Bacon, M.C. Johnson, J. Chem. Ecol. 1990, 16, 3301-3315.

[3] E.N. Dubis, L.B. Brattsten, L.B. Dungan, Effects of the Endophyte - Associated Alkaloids Peramine on Southern Armworm Microsomal Cytochrome P450, ACS Symposium Series Molecular mechanism of Insecticide Resistance Diversity Among Insects, ACS, Washington, DC, 1992.

[4] F.F. Fannin, L.P. Busch, M.R. Siegel, D.D. Rowan, J. Chromatogr. 1990, 503, 288-292.

[5] H.W. Zanhg, Y.Ch. Song, R.X. Tan, Nat. Prod. Rep. 2006, 23, 753-771.

[6] J. Krauss, S.A. Härri, L. Busch, R. Husi, L. Bigler, S.A. Power, C.B. Müller, Functional Ecol. 2007, 21, 107-116.

[7] A. Koulman, G.A. Lane, M.J. Christensen, K. Fraser, B.A. Tapper, Phytochem. 2007, 68, 355-364.

[8] E. Dubis, A. Dubis, J. Nawrot, Z. Winiecki, J. Popławski, Effects of the Endophyte-Associated Alkaloids Peramine and its Analogues on Selected Storage Pests, Proceedings of the $1^{\text {st }}$ International Conference on Insects, Insects - Chemical, Physiological and Environmental Aspects, Lądek-Zdrój, Poland, University of Wrocław, 1995, pp 306-310.

[9] M.S.J. Simmonds, Phytochem. 1998, 49, 1183-1190.

[10] Y. Ikemoto, Y. Matsuzawa, J. Mizutani, Pestic. Biochem. Physiol. 1995, 52, 6070.

[11] M.J. Spiering, E. Davies, B.A. Tapper, J. Schmidt, G.A. Lane, J. Agric. Food Chem. 2002, 50, 5856-5862.

[12] C.L. Schardl, A. Leutchman, M.J. Spiering, Annual Review of Plant Biology 2004, 55, 315-340.

[13] A.T. Dubis, A. Łapiński, Vibr. Spectr. 2009, 49, 265-273.

[14] E.J. Baerends, G. Ricciardi, A. Rosa, S.J.A. van Gisbergen, Coord. Chem. Rev. 2002, 230, 5-27.

[15] R.H. Wilson, H.S. Tapp, Trends Anal. Chem. 1999, 18, 85-94.

[16] D.I. Ellis, D. Broadhurst, R. Goodacre, Anal. Chim. Acta 2004, 514, 193-204.

[17] K.B. Lipkowitz, R. Larter, T.R. Cundari (editors), Reviews in Computational Chemistry, Volume 20, John Wiley\&Sons, Inc, 2004.

[18] J. Kohanoff, in Electronic structure calculations for solids and molecules. Theory and computational methods, Cambridge, UK, 2006.

[19] K. Burke, J. Werschnik, E.K.U. Gross, J. Chem. Phys. 2005, 123, 62206-62209.

[20] E. Runge, E.K.U. Gross, Phys. Rev. Lett. 1984, 52, 997-1000.

[21] M.J. Frisch, G.W. Trucks, H.B. Schlegel, G.E. Scuseria, M.A. Robb, J.R. Cheeseman, J.A. Montgomery, Jr.,T. Vreven, K.N. Kudin, J.C. Burant, J.M. Millam, S.S. Iyengar, J. Tomasi, V. Barone, B. Mennucci, M. Cossi, G. Scalmani, N. Rega, G.A. Petersson, H. Nakatsuji, M. Hada, M. Ehara, K. Toyota, R. Fukuda, J. Hasegawa, M. Ishida, T. Nakajima, Y. Honda, O. Kitao, H. Nakai, M. Klene, X. Li, J.E. Knox, H.P. Hratchian, J.B. Cross, C. Adamo, J. 
Jaramillo, R. Gomperts, R.E. Stratmann, O. Yazyev, A.J. Austin, R. Cammi, C. Pomelli, J.W. Ochterski, P.Y. Ayala, K. Morokuma, G.A. Voth, P. Salvador, J.J. Dannenberg, V.G. Zakrzewski, S. Dapprich, A.D. Daniels, M.C. Strain, O. Farkas, D.K. Malick, A.D. Rabuck, K. Raghavachari, J.B. Foresman, J.V. Ortiz, Q. Cui, A.G. Baboul, S. Clifford, J. Cioslowski, B.B. Stefanov, G. Liu, A. Liashenko, P. Piskorz, I. Komaromi, R.L. Martin, D.J. Fox, T. Keith, M.A. AlLaham, C.Y. Peng, A. Nanayakkara, M. Challacombe, P.M.W. Gill, B. Johnson, W. Chen, M.W. Wong, C. Gonzalez, and J.A. Pople, Gaussian 03, Revision B.03, Gaussian, Inc., Pittsburgh PA 2003.

[22] A.D. Becke, Phys. Rev. A 1988, 38, 3098-3100.

[23] A.D. Becke, J. Chem. Phys. 1993, 98, 5648-5652.

[24] C. Lee, W. Yang, R.G. Parr, Phys. Rev. B 1988, 37, 785-789.

[25] A.P. Scott. L. Radom, J. Phys. Chem. 1996, 100, 16502-16513.

[26] R. Sun. J. Yao, S. Li, R Gu., Vib. Spectr. 2008, 47, 38-43.

[27] D. Michalska, R. Wysokinski, Chem. Phys. Letters 2005, 403, 211-217.

[28] P.L. Polavarapu, J. Phys. Chem. 1990, 94, 8106-8112.

[29] F. Taddei, J. Mol. Struct. (Theochem) 2001, 544, 141-150.

[30] T.J. Dwyer, P.G. Jasien, J. Mol. Struct. (Theochem) 1996, 363, 139-150.

[31] R. Benassi, C. Bertarini, E. Kleinpeter, F. Taddei, S. Thomas, J. Mol. Struct. (Theochem) 2000, 498, 201-215.

[32] R. Benassi, E. Kleinpeter, F. Taddei, J. Mol. Struct. (Theochem) 2001, 541, 101110.

[33] G.A. Jeffrey, W. Saenger, in Hydrogen Bonding in Biological Structures, Springer-Verlag, Berlin Heidelberg, 1991.

[34] S. Grabowski, in Hydrogen Bonding - New Insights, Edited by S. Grabowski, Springer, Dordrecht, 2006.

[35] L. Sobczyk, S.J. Grabowski, T.M. Krygowski, Chem. Rev. 2005, 105, 35133560.

[36] F. Furche, D. Rappoport, in: M. Olivucci (Ed.), in Computational Photochemistry, 16, Elsevier, Amsterdam, 2005.

[37] L. Bernasconi, M. Sprik, R. Hutter, Chem. Phys. Lett. 2004, 394, 141-151.

[38] C.J. Jamorski, M.E. Casida, J. Phys. Chem. B 2004, 108, 7132-7141.

[39] A. Dreuw, M. Head-Gordon, J. Am. Chem. Soc. 2004, 126, 4007-4016.

[40] D.J. Tozer, J. Chem. Phys. 2003, 119, 12697-12699.

[41] C.J. Jamorski, H.P. Luthi, J. Chem. Phys. 2003, 119, 12852-12865.

[42] J. Fabian, Theor. Chem. Acc. 2001, 106, 199-205.

[43] O. Gritsenko, E.J. Baerends, J. Chem. Phys. 2004, 121, 655-660.

[44] N.S. Bayliss, E.G. McRae, J. Phys. Chem. 1954, 58, 1002-1006.

[45] N. Mataga, T. Kubato, in Molecular Interactions and Electronic Spectra, Marcel Dekker, New York, 1970.

[46] Y. Ooshika, J. Phys. Soc. Japan 1954, 9, 594-602.

[47] E.G. McRae, J. Phys. Chem. 1957, 61, 562-572. 
[48] H.C. Longuet-Higgins, J.A. Pople, J. Chem. Phys. 1957, 27, 192-201.

[49] N. Scott, G.K. Vemulapalli, Spectrochimica Acta A 2000, 56, 2373-2377.

[50] S.E. Blanco, J.J. Silber, F.H. Ferretti, J. Mol. Struct, (Theochem) 2002, 582, 91105.

[51] G.A. Jeffrey, in An Introduction to Hydrogen Bonding, Oxford University Press, 1997. 
Table 1. Selected optimized geometrical parameters of three different forms of peramine calculated at B3LYP/6-311++G(d,p) level. Atom numbering is given in Fig. 2

\begin{tabular}{lccc}
\hline & I & II & III \\
\hline Bond lengths $(\AA)$ & & & \\
\hline C1-C3 & 1.411 & 1.411 & 1.411 \\
C3-C5 & 1.387 & 1.387 & 1.387 \\
C5-N6 & 1.390 & 1.389 & 1.390 \\
N6-C2 & 1.368 & 1.369 & 1.369 \\
C2-C1 & 1.386 & 1.386 & 1.386 \\
C5-C9 & 1.449 & 1.448 & 1.449 \\
C9-O11 & 1.224 & 1.224 & 1.224 \\
C9-N12 & 1.413 & 1.412 & 1.413 \\
N12-C16 & 1.465 & 1.466 & 1.466 \\
N12-C13 & 1.403 & 1.405 & 1.403 \\
C13-C10 & 1.350 & 1.349 & 1.350 \\
C10-N6 & 1.390 & 1.391 & 1.390 \\
C13-C15 & 1.509 & 1.511 & 1.509 \\
C15-C17 & 1.542 & 1.531 & 1.542 \\
C17-C23 & 1.528 & 1.530 & 1.528 \\
C23-N26 & 1.459 & 1.459 & 1.459 \\
N26-C29 & 1.386 & 1.387 & 1.386 \\
C29-N32 & 1.281 & 1.281 & 1.281 \\
C29-N31 & 1.403 & 1.403 & 1.403 \\
\hline Dihedral angle $\left(^{\circ}\right)$ & & & \\
\hline C10-C13-C15-C17 & 95.8 & 1.6 & 276.3 \\
\hline
\end{tabular}


Table 2. B3LYP/6-31++G(d,p) total energies ( $E$; Hartree/molecule), relative energies ( $\left.\triangle E E-E_{I} ; \mathrm{kcal} / \mathrm{mol}\right)$, and dipole moment ( $\mu$; Debye) for the peramine and the transition states connecting the minima

\begin{tabular}{cccc}
\hline Structure & $E$ & $\Delta E$ & $\mu$ \\
\hline I & -816.55881 & 0.0 & 4.9 \\
TS3 & -816.55411 & 2.95 & 6.1 \\
II & -816.55673 & 1.30 & 6.6 \\
TS2 & -816.55412 & 2.94 & 6.2 \\
III & -816.55687 & 1.22 & 5.3 \\
TS1 & -816.47909 & 50.03 & 3.7 \\
\hline
\end{tabular}


Table 3. B3LYP/6-31++G(d,p) total energies ( $E$; Hartree/molecule), relative energies ( $\left.\triangle E=E_{I I} E_{I} ; \mathrm{kcal} / \mathrm{mol}\right)$, and dipole moment $(\mu ;$ Debye) for flat (II) and bent (I) conformers of compounds 1-4

\begin{tabular}{cccc}
\hline Compound & $E$ & $\Delta E$ & $M$ \\
\hline $\mathbf{1}(\mathrm{I})$ & -816.73040 & \multirow{2}{*}{0.28} & 4.89 \\
$\mathbf{1}(\mathrm{II})$ & -816.72995 & & 6.72 \\
\hline $\mathbf{2}(\mathrm{I})$ & -667.86361 & \multirow{2}{*}{0.15} & 6.14 \\
$\mathbf{2}(\mathrm{II})$ & -667.86338 & & 4.39 \\
\hline $\mathbf{3}(\mathrm{I})$ & -1072.12146 & \multirow{2}{*}{0.18} & 4.49 \\
$\mathbf{3}(\mathrm{II})$ & -1072.12117 & & 2.97 \\
\hline $\mathbf{4}(\mathrm{I})$ & -612.49943 & \multirow{2}{*}{0.19} & 5.31 \\
$\mathbf{4}(\mathrm{II})$ & -612.49913 & & 5.09 \\
\hline
\end{tabular}


Table 4. Selected optimized geometrical parameters of peramine (1) and its derivatives (2-4) for I conformation calculated at B3LYP/6-311++G(d,p) level. Atom numbering is given in Fig. 2

\begin{tabular}{lcccc}
\hline & $\mathbf{1}$ & $\mathbf{2}$ & $\mathbf{3}$ & $\mathbf{4}$ \\
\hline Bond lengths $(\AA)$ & & & & \\
\hline C13-C10 & 1.350 & 1.350 & 1.350 & 1.350 \\
C9-O11 & 1.224 & 1.224 & 1.224 & 1.224 \\
N12-C16 & 1.465 & 1.466 & 1.465 & 1.466 \\
C13-C15 & 1.509 & 1.509 & 1.509 & 1.509 \\
C10-H14 & 1.079 & 1.079 & 1.079 & 1.079 \\
N6-C2 & 1.368 & 1.369 & 1.369 & 1.368 \\
C2-C1 & 1.386 & 1.386 & 1.386 & 1.386 \\
N12-C13 & 1.403 & 1.403 & 1.402 & 1.403 \\
C2-H7 & 1.078 & 1.078 & 1.078 & 1.078 \\
C1-H4 & 1.079 & 1.079 & 1.079 & 1.079 \\
C3-H8 & 1.078 & 1.078 & 1.078 & 1.078 \\
\hline Dihedral angle $\left(^{\circ}\right)$ & & & & \\
\hline C10-C13-C15-C17 & 95.8 & 97.0 & 95.9 & 96.2 \\
\hline
\end{tabular}


Table 5. Orbital energy $(\mathrm{eV})$ of the frontier orbitals of three different rotameric forms of peramine calculated at B3LYP/6-311++G(d,p) level.

\begin{tabular}{lccc}
\hline \multirow{2}{*}{ Molecular Orbital } & \multicolumn{3}{c}{$E(\mathrm{eV})$} \\
\cline { 2 - 4 } & $\mathrm{I}$ & $\mathrm{II}$ & $\mathrm{III}$ \\
\hline LUMO+8 & 0.52 & 0.49 & 0.49 \\
LUMO+7 & 0.35 & 0.27 & 0.38 \\
LUMO+6 & 0.19 & 0.16 & 0.08 \\
LUMO+5 & 0.00 & 0.08 & 0.05 \\
LUMO+4 & -0.19 & -0.22 & -0.19 \\
LUMO+3 & -0.27 & -0.24 & -0.33 \\
LUMO+2 & -0.38 & -0.41 & -0.46 \\
LUMO+1 & -0.68 & -0.76 & -0.60 \\
LUMO & -0.82 & 0.79 & -0.82 \\
HOMO & -5.74 & -5.71 & -5.74 \\
HOMO-1 & -5.74 & -6.29 & -6.31 \\
HOMO-2 & -6.67 & -6.72 & -6.67 \\
HOMO-3 & -7.26 & -7.24 & -7.24 \\
\hline
\end{tabular}


Table 6. HOMO-LUMO energy gaps of the molecules under study for the bent conformation (I) calculated at B3LYP/6-311++G(d,p) level.

\begin{tabular}{cccc}
\hline Compound & HOMO $(\mathrm{eV})$ & LUMO $(\mathrm{eV})$ & $\Delta E(\mathrm{eV})$ \\
\hline $\mathbf{1}$ & -5.742 & -0.816 & 4.926 \\
$\mathbf{2}$ & -5.688 & -0.789 & 4.898 \\
$\mathbf{3}$ & -5.878 & -0.925 & 4.953 \\
$\mathbf{4}$ & -5.688 & -0.789 & 4.898 \\
\hline
\end{tabular}


Table 7. Excitation energies ( $\triangle E$ in $\mathrm{eV})$, oscillator strengths $f$ of the singlet-singlet transitions of peramine (1) and its derivatives (2-4).

\begin{tabular}{|c|c|c|c|c|c|c|c|c|c|}
\hline & \multicolumn{3}{|c|}{ Experiment $\Delta E(\mathrm{eV})$} & \multicolumn{6}{|c|}{ Theory $(\mathrm{eV})$} \\
\hline & \multirow[t]{2}{*}{$\mathrm{KBr}$} & \multirow[t]{2}{*}{$\mathrm{H}_{2} \mathrm{O}$} & \multirow[t]{2}{*}{$\mathrm{C}_{2} \mathrm{H}_{5} \mathrm{OH}$} & \multicolumn{2}{|c|}{$\begin{array}{c}T D-B 3 L Y P / \\
311++G(d, p)\end{array}$} & \multicolumn{2}{|c|}{$\begin{array}{c}T D-P B E 0 / \\
311++G(d, p)\end{array}$} & \multicolumn{2}{|c|}{$\begin{array}{c}T D-P B E 0 / \\
311++G(d, p) \\
P C M(\text { ethanol })\end{array}$} \\
\hline & & & & $\Delta E$ & $f$ & $\Delta E$ & $f$ & $\Delta E$ & $f$ \\
\hline \multirow{3}{*}{1} & 4.25 & 4.30 & 4.36 & 4.41 & 0.1842 & 4.57 & 0.1968 & 4.42 & 0.2349 \\
\hline & \multirow{2}{*}{5.44} & \multirow{2}{*}{5.41} & \multirow{2}{*}{5.39} & 5.48 & 0.3184 & 5.67 & 0.5098 & 5.48 & 0.4732 \\
\hline & & & & 5.58 & 0.2496 & 5.80 & 0.1011 & 5.66 & 0.2671 \\
\hline \multirow{3}{*}{2} & 4.30 & & 4.35 & 4.42 & 0.1595 & 4.57 & 0.1829 & 4.42 & 0.2265 \\
\hline & \multirow{2}{*}{5.47} & & \multirow{2}{*}{5.40} & 5.54 & 0.4838 & 5.62 & 0.1565 & 5.47 & 0.4941 \\
\hline & & & & 5.56 & 0.1366 & 5.71 & 0.4190 & 5.67 & 0.3110 \\
\hline \multirow{3}{*}{3} & 4.37 & 4.31 & 4.36 & 4.44 & 0.1624 & 4.59 & 0.1786 & 4.44 & 0.2278 \\
\hline & \multirow{2}{*}{5.44} & \multirow{2}{*}{5.42} & \multirow{2}{*}{5.39} & 5.37 & 0.1944 & 5.62 & 0.3162 & 5.46 & 0.5345 \\
\hline & & & & 5.60 & 0.3186 & 5.68 & 0.2084 & 5.64 & 0.2002 \\
\hline \multirow{3}{*}{4} & 4.27 & & & 4.41 & 0.1695 & 4.57 & 0.1819 & 4.42 & 0.2248 \\
\hline & \multirow{2}{*}{5.39} & & & 5.44 & 0.0282 & 5.68 & 0.4530 & 5.48 & 0.5193 \\
\hline & & & & 5.53 & 0.5422 & 5.73 & 0.1436 & 5.67 & 0.3244 \\
\hline
\end{tabular}




\section{Figure captions}

Figure 1. Reaction coordinate for rotation aliphatic chain about the C13-C15 single bond in peramine (see Fig. 2)

Figure 2. Peramine molecule with atom numbering; $\tau$ means the torsion angle $(\mathrm{C} 10$, $\mathrm{C} 13, \mathrm{C} 15, \mathrm{C} 17)$

Figure 3. Rotamer states I, II, III and transition states TS1, TS2, and TS3 of peramine (1)

Figure 4. Raman spectra of peramine (1) recorded with different excitations: 633, 568, 514, and $488 \mathrm{~nm}$ (upper panel). Theoretical Raman spectra calculated for three conformations I, II, and III at the theory level B3LYP/6-311++G(d,p) (lower panel); the spectra were scaled using the 0.98 scaling factor. Note: $v$ means stretching and $\delta$ bending vibrations

Figure 5. Sketch of the frontier orbitals of peramine computed at the B3LYP/6$311++\mathrm{G}(\mathrm{d}, \mathrm{p})$ theory level

Figure 6. Absorption spectra of peramine recorded in $\mathrm{KBr}$ pellets and in water and ethanol solutions (upper panels); the band positions were obtained by fitting the spectra by Gaussian curves (dotted lines). The calculated electronic transitions performed at the theory level TD-B3LYP/6-311++G(d,p) for bent conformation (I) (the lowest panel)

Figure 7. Absorption spectra of peramine derivatives 2-4 recorded in $\mathrm{KBr}$ pellets and in water and ethanol solutions (upper panels). The calculated electronic transitions performed for bent and flat conformations for chloro derivative at the theory level TDB3LYP/6-311++G(d,p) (the lowest panel) 


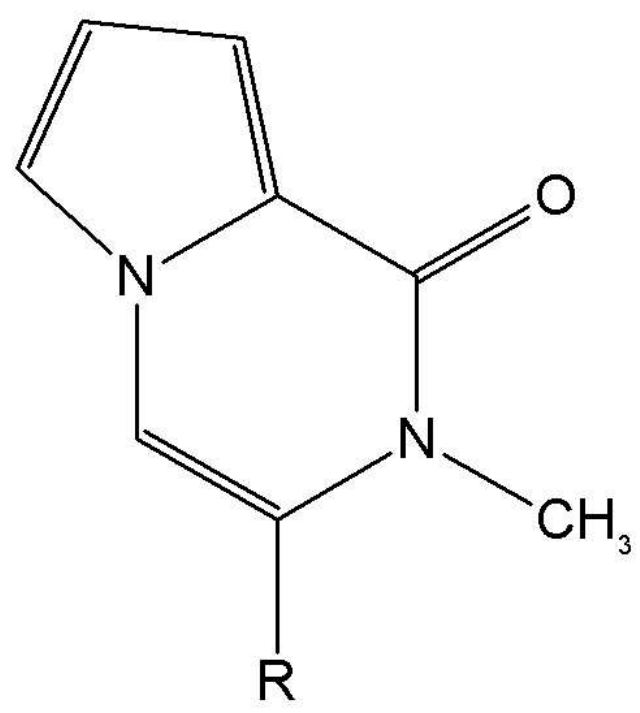

\section{Peramine (1) $\quad \mathrm{R}=-\mathrm{CH}_{2} \mathrm{CH}_{2} \mathrm{CH}_{2} \mathrm{NHCNHNH}_{2}$}

Amino derivative (2) $\mathrm{R}=-\mathrm{CH}_{2} \mathrm{CH}_{2} \mathrm{CH}_{2} \mathrm{NH}_{2}$

Chloro derivative (3) $\mathrm{R}=-\mathrm{CH}_{2} \mathrm{CH}_{2} \mathrm{CH}_{2} \mathrm{Cl}$

Alkyl derivative (4) $\mathrm{R}=-\mathrm{CH}_{2} \mathrm{CH}_{2} \mathrm{CH}_{3}$ 


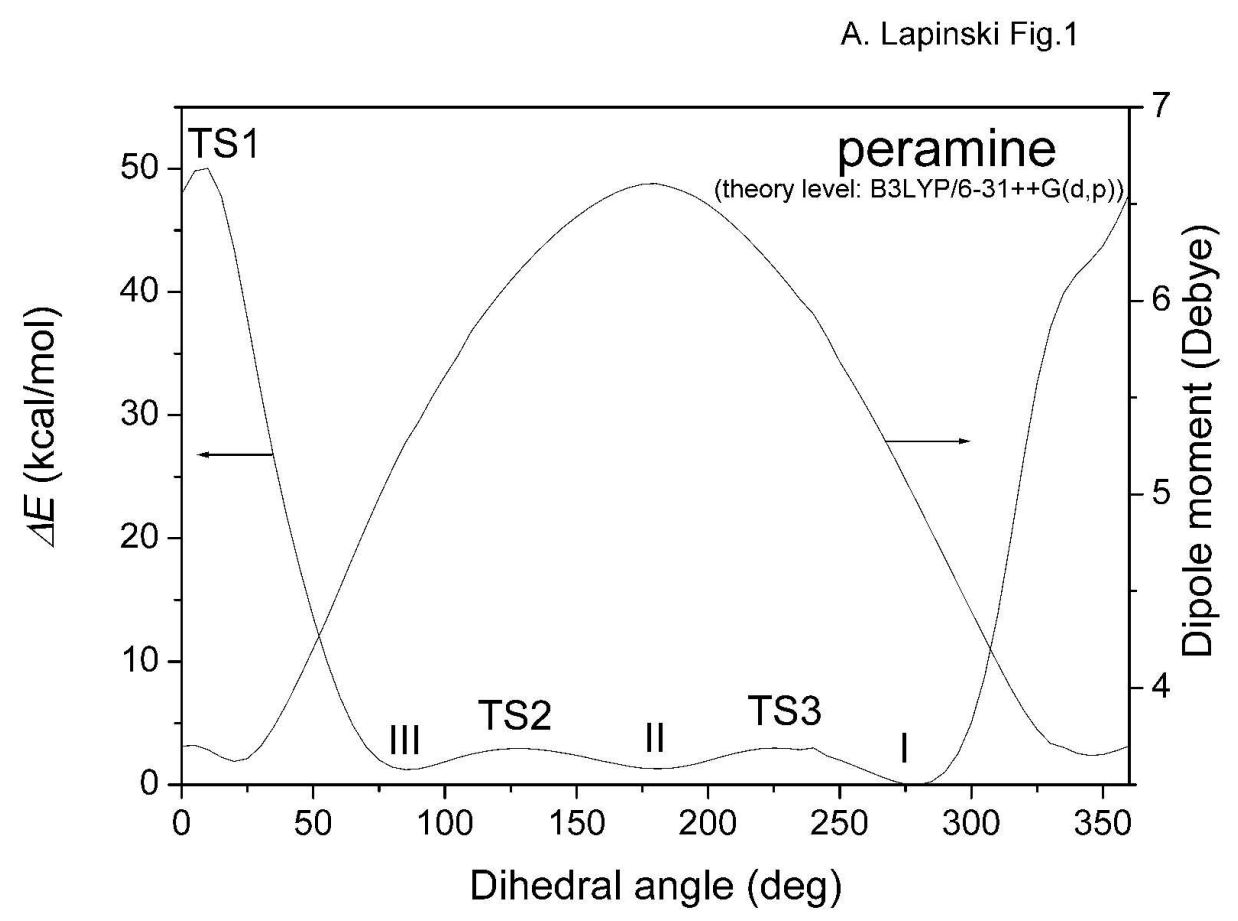

Figure 1. Reaction coordinate for rotation aliphatic chain about the $\mathrm{C} 13-\mathrm{C} 15$ single bond in peramine (see Fig. 2)

$1802 \times 1259 \mathrm{~mm}(96 \times 96$ DPI $)$ 


\section{A. Lapinski Fig. 2}

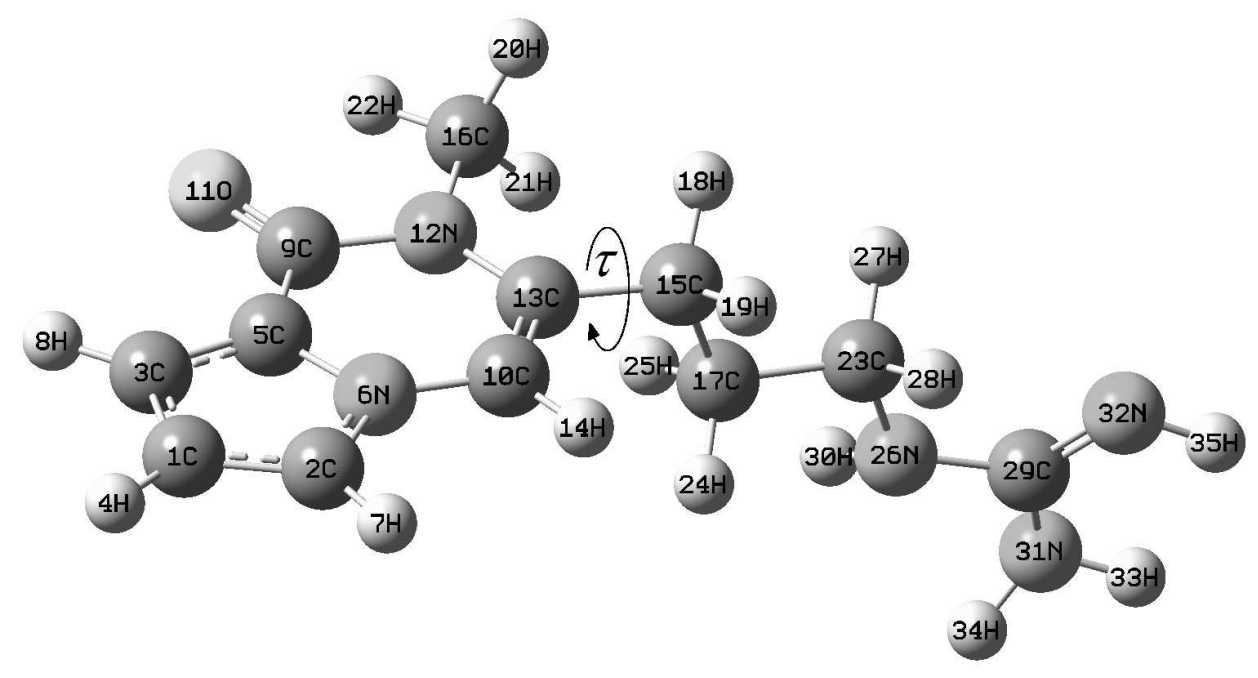

Peramine molecule with atom numbering; T means the torsion angle (C10, C13, C15, C17) $842 \times 701 \mathrm{~mm}(96 \times 96 \mathrm{DPI})$ 
Figure 3. Rotamer states I, II, III and transition states TS1, TS2, and TS3 of peramine (1) $899 \times 476 \mathrm{~mm}(96 \times 96 \mathrm{DPI})$ 
Figure 4. Raman spectra of peramine (1) recorded with different excitations: 633, 568, 514, and $488 \mathrm{~nm}$ (upper panel). Theoretical Raman spectra calculated for three conformations I, II, and III at the theory level B3LYP/6-311++G(d,p) (lower panel); the spectra were scaled using the 0.98 scaling factor. Note: ni means stretching and $\delta$ bending vibrations $201 \times 288 \mathrm{~mm}(600 \times 600 \mathrm{DPI})$ 
Figure 5 . Sketch of the frontier orbitals of peramine computed at the B3LYP/6-311++G(d,p) theory level $889 \times 608 \mathrm{~mm}(96 \times 96$ DPI) 


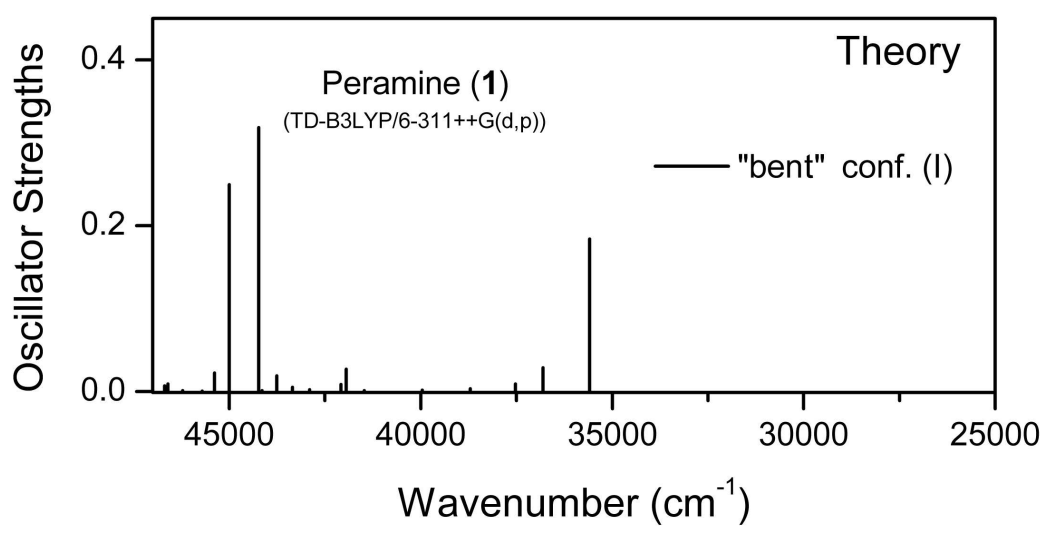

Figure 6. Absorption spectra of peramine recorded in $\mathrm{KBr}$ pellets and in water and ethanol solutions (upper panels); the band positions were obtained by fitting the spectra by Gaussian curves (dotted lines). The calculated electronic transitions performed at the theory level TD-B3LYP/6-311++G(d,p) for bent conformation (I) (the lowest panel) $635 \times 868 \mathrm{~mm}(96 \times 96 \mathrm{DPI})$ 


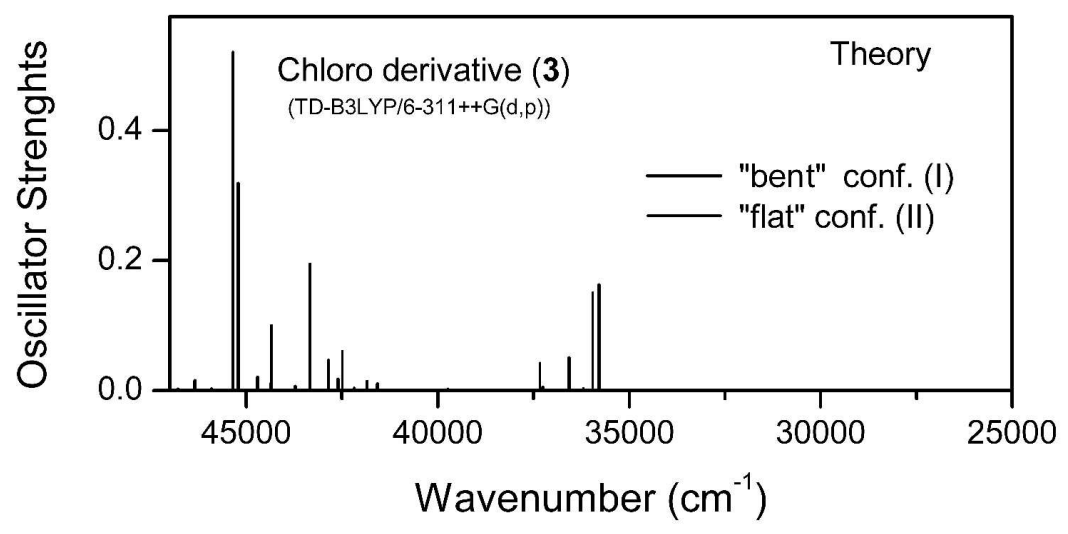

Figure 7. Absorption spectra of peramine derivatives 2-4 recorded in $\mathrm{KBr}$ pellets and in water and ethanol solutions (upper panels). The calculated electronic transitions performed for bent and flat conformations for chloro derivative at the theory level TD-B3LYP/6-311++G(d,p) (the lowest panel) $1270 \times 1735 \mathrm{~mm}(96 \times 96 \mathrm{DPI})$ 